\title{
316 LEUCINE-RICH REPEATS AND IMMUNOGLOBULIN-LIKE DOMAINS PROTEIN 1 IS HIGHLY EXPRESSED ON TUMOR-INFILTRATING T CELLS AND MAY PLAY A ROLE IN MODULATING T CELL ACTIVATION
}

Li Wang, Timothy Chan, Cassandra Gilmour, Keman Zhang, Tyler Alban, Hieu Ta*, Hieu Ta. Cleveland Clinic, Cleveland, OH, USA

Background Immune checkpoint receptors (ICRs) regulate $\mathrm{T}$ cell-mediated immune responses to self - and neo-antigens, thereby play an important role in the development of autoimmunity and immune responses to infections. In cancer, ICRs such as PD-1 and CTLA4 impair immuno-surveillance against neoplastic cells, while other costimulatory molecules like ICOS, OX40 could augment the immune responses. Immunotherapy that targets either inhibitory or costimulatory immune checkpoint have become the breakthrough therapy for cancer treatment. However, the efficacy of this class of therapy is limited to a fraction of patients, pointing the need to overcome non-redundant immuno-inhibitory pathways or discover new immuno-costimulatory pathways.

Methods Examining surface proteins that are highly expressed in various mouse tumors by flow cytometry and immunohistochemistry

Results This study identified the expression of Leucine-rich repeats and immunoglobulin-like domains protein 1 (LRIG1) in activated $\mathrm{CD} 4+$ and $\mathrm{CD} 8+\mathrm{T}$ cells. LRIG1 is a type-I transmembrane protein with 1092 amino acids $(120 \mathrm{kD})$ and ubiquitous tissue expression patterns. We show that LRIG1 expression is not detectable on naïve CD4+ and CD8+ murine $\mathrm{T}$ cells but is induced upon $\mathrm{T}$ cell receptor (TCR) stimulation in vitro, and in tumor-infiltrating lymphocytes (TILs) from multiple murine models. We also detected LRIG1 expression on CD4+ and CD8+ TILs in several types of cancer patients such as non-small-cell lung cancer, breast cancer, melanoma, B cell lymphoma.

Conclusions LRIG1 was originally identified as a tumor suppressor gene in a variety of tissues, but its expression and function have not been explored in immune cells. Our data suggest that LRIG1 is highly expressed in TILs in multiple cancer types and might play a role in regulating the antitumor immune response

Ethics Approval Our studies have been approved by the ethics committee of the Cleveland Clinic.

Consent Written informed consent was obtained from the patient for publication of this abstract and any accompanying images. A copy of the written consent is available for review by the Editor of this journal.

http://dx.doi.org/10.1136/jitc-2021-SITC2021.316 